\title{
LA CALIDAD DE VIDA POR DEPARTAMENTOS, PROVINCIAS Y REGIONES EN EL PRIMER CENSO NACIONAL (1869) ${ }^{1}$
}

\author{
Quality of life by provinces, regions and municipalities, in 1869 first \\ argentina's national census
}

\author{
Guillermo Velázquez* \\ https://orcid.org/0000-0003-0892-6572 \\ Hernán Otero** \\ https://orcid.org/0000-0001-5180-3987
}

\section{Resumen}

El presente artículo analiza desde la Geografía y la Historia las variables más relevantes, y su contexto, para mostrar la calidad de vida en el Primer Censo Nacional de población de la Argentina (1869). Para esto, se consideran variables habitualmente soslayadas, en particular las vinculadas con educación, salud y condiciones de vivienda. El objetivo central es proponer un índice de calidad de vida que permita, al mismo tiempo, utilizar las fuentes del período y posibilitar futuras comparaciones diacrónicas y con otros contextos. Desde el punto de vista metodológico, se utilizan herramientas estadísticas y Sistemas de Información Geográfica (SIG), aplicadas a las unidades espaciales de mayor nivel de desagregación disponibles (departamentos/partidos). Los resultados muestran que el índice de calidad de vida tiene un alto grado de desigualdad y suministran aportes relevantes para investigaciones posteriores.

$<$ Calidad de vida $><$ Desigualdad $><$ Censo de 1869 $><$ Departamentos $>$

\begin{abstract}
This article analyzes the First National Population Census of Argentina (1869) from geographical and historical perspectives. We studied its most relevant variables and also their context in order to show the quality of life at that time. Some usually disregarded variables are considered, particularly those related to education, health and housing conditions. The main objective of this work is to propose an index of quality of life that allows us to analyze the sources of the period and, at the same time, to enable future diachronic comparisons with another contexts. From the methodological point of view, statistical techniques and Geographic Information Systems (GIS) are applied to these spatial units with the highest available disaggregating level (districts/municipalities). Results show high degree of inequality all over the country and provinces inside. They also provide relevant contributions for subsequent researches.
\end{abstract}

$<$ Quality of life $><$ Inequality $><$ Census of $1869><$ Municipalities $>$

Recibido: 06/09/2018//Aceptado: 14/11/2018

${ }_{1}$ El presente artículo forma parte del Proyecto de Unidades Ejecutoras del Consejo Nacional de Investigaciones Científicas y Técnicas (CONICET) núm. 229-201601-00003 titulado Configuración y reconfiguración socio-territorial de la Argentina en tiempos del Bicentenario. Se presentó una versión preliminar en las XXVI Jornadas de Historia Económica, Santa Rosa, septiembre de 2018.

* Instituto de Geografía, Historia y Ciencias Sociales (IGEHCS), Investigador Superior del Consejo Nacional de Investigaciones Científicas y Técnicas (CONICET), Argentina, gvelaz@fch.unicen.edu.ar

** Instituto de Geografía, Historia y Ciencias Sociales (IGEHCS), Investigador Principal del Consejo Nacional de Investigaciones Científicas y Técnicas (CONICET), Argentina, hernan.otero@conicet.gov.ar 
Velázquez y Otero. Calidad de vida por departamentos, provincias y regiones en el primer censo nacional (1869)

\section{Introducción}

Medir la calidad de vida requiere de un indicador que sintetice un conjunto de situaciones socio-demográficas, económicas y ambientales relevantes para reflejar las condiciones de la población en ese tiempo y lugar determinados. Esto le otorga interés para la formulación de políticas públicas y en los casos históricos, como el que abordamos ahora, permite también la comprensión del funcionamiento social desde perspectiva de evolución histórica. Nos remontamos a la década de los noventa como impulso inicial de estudios de calidad de vida en el contexto latinoamericano (Camargo Mora, 1996; Delgado y Méndez, 1996; Olave y Bodini, 1995; Velázquez y García, 1999), y la valiosa información que se obtiene de ellos justifica su crecimiento, desde entonces y de manera significativa también para el caso argentino (Lucero et al, 2007; Mikkelsen et al, 2013; Velázquez, 2001; Velázquez, 2008; Velázquez et al, 2014). A pesar de las enormes dificultades empíricas que presentan los datos, especialmente los previos al período estadístico, el tema ha recibido también atención por parte de los historiadores. ${ }^{2}$ Las discusiones se centraron en los cambios producidos por la progresiva incorporación a la economía atlántica a lo largo del siglo XIX y, sobre todo, en sus efectos en términos de disparidades regionales y de equidad. Si bien el carácter sincrónico del presente estudio nos impide comparaciones con los períodos previos, las discusiones mencionadas suministran insumos de interés para futuras comparaciones del índice propuesto. Cabe consignar que, al igual que en la producción mencionada (en particular, Hora, 2010) utilizaremos el término calidad de vida en un sentido amplio, es decir como sinónimo de otros como los de condiciones de vida o bienestar. Partiendo de este contexto, el presente estudio se propone analizar la calidad de vida de la población durante el Primer Censo Nacional de la República Argentina levantado en 1869, con el propósito de suministrar un panorama general de las diferencias socio-espaciales que caracterizaban al país hacia mediados del siglo XIX.

La utilización del censo de población no resulta casual, ya que es la única fuente que permite alcanzar una cobertura universal de la totalidad del territorio en cada momento histórico. Cabe consignar, asimismo, que si bien los censos del período 1869-1914 han sido visitados con frecuencia, los trabajos realizados han priorizado su uso demográfico (por ejemplo, Recchini de Lattes y Lattes, 1975; Torrado, 2007, por mencionar sólo dos obras de referencia) o su análisis como fuente en segundo grado, abocada al estudio de las categorías de su diseño conceptual o los aspectos políticos e institucionales propios de la socio-historia de la estadística (González Bollo, 2014; Massé, 2003; Otero, 2006). A pesar de sus aportes, estas corrientes no agotan la riqueza de los relevamientos históricos, ya que dejan de lado un amplio conjunto de variables de interés, en particular las relativas a la vivienda y la salud. La necesidad de

\footnotetext{
2 La bibliografía histórica es bastante amplia. Ver, por ejemplo, los trabajos de Salvatore (en particular, 1998) sobre el bienestar biológico medido a través de la evolución de las estaturas, indicador por cierto no exento de dificultades interpretativas, y los estudios sobre riqueza material en base a fuentes judiciales, económicas y fiscales (por ejemplo, Gelman, 2011; Guzmán, 2012; Gelman y Santilli, 2018). Para síntesis de los debates sobre la evolución del bienestar y la equidad en la etapa previa a 1930 ver Míguez (2008, pp. 138-143) y Hora (2010, pp. 87-93//154-164).
} 
multidisciplinariedad y multidimensionalidad, y los pocos avances logrados al respecto hasta el momento, se resumen en el dossier de Santilli (2016). Desde el punto de vista metodológico, el trabajo se apoya en el uso de Sistemas de Información Geográfica (SIG), para el análisis espacial de las dimensiones, y variables relevantes, y para la elaboración de un índice de calidad de vida (ICV) acorde con la información disponible y las características del período histórico considerado. Un primer elemento a destacar de la propuesta es la utilización de la escala de mayor nivel de desagregación territorial posible (denominada departamentos o partidos según la provincias) ${ }^{3}$, tarea que exige el relevamiento, procesamiento y digitalización de la compleja cartografía de un período caracterizado, hasta fines de siglo, por la expansión del Estado nacional hacia las áreas de frontera y por la concomitante creación de partidos y departamentos, muchos de ellos de gran tamaño, llamados a subdividirse o a desaparecer en etapas posteriores. Estas dificultades explican que la producción histórica realizada hasta el momento se haya concentrado en unidades de mayor tamaño, como las provincias y regiones (por ejemplo, Salvatore, 1998; Otero, 2004). Un segundo elemento de interés es la elaboración de un índice compuesto, que sintetiza variables de todas las dimensiones relevantes (educación, salud, vivienda), lo que permite reducir los problemas de captación derivados de cada una de ellas y proporcionar una imagen más completa y multidimensional que las basadas en un único indicador.

Dados los problemas mencionados, y la posibilidad de aleatoriedad estadística en función de la reducida población de algunas unidades, el objetivo central consiste en suministrar un panorama comparativo de conjunto atento a las diferencias inter, pero sobre todo, intra provinciales, más que al análisis de unidades específicas, algunas de las cuales pueden presentar comportamientos atípicos que apelan por estudios específicos. Los resultados obtenidos sirven, además, como base para comparaciones diacrónicas con las imágenes obtenidas a partir de los censos más recientes. Va de suyo que tales comparaciones no pueden basarse exactamente en los mismos indicadores (inexistentes a nivel departamental para períodos antiguos, como ocurre con el caso emblemático de la mortalidad infantil) pero sí en indicadores equivalentes que apunten a medir las mismas dimensiones subyacentes. En síntesis, la proposición de un índice de calidad de vida para el período analizado, la comparación intra caso, y la confluencia interdisciplinaria entre Geografía e Historia constituyen los objetivos centrales de la propuesta. La comparación con momentos posteriores, y profundizaciones en el modelo estadístico explicativo, superan el espacio de este artículo y serán tratados en futuros trabajos.

\section{La fuente y su contexto}

Debido a que el censo constituye la fuente de base de la propuesta, importa reseñar algunos aspectos centrales de su realización para contextualizar adecuadamente sus ventajas y límites. El Primer Censo de la República Argentina, dirigido por el

\footnotetext{
3 La única excepción es la ciudad de Buenos Aires, que es analizada como un todo en el presente estudio, si bien el censo presenta información desagregada por secciones de policía. La elaboración de la cartografía se basó en el trabajo de Cacopardo (1967).
} 
Velázquez y Otero. Calidad de vida por departamentos, provincias y regiones en el primer censo nacional (1869)

Superintendente Diego Gregorio de la Fuente durante la presidencia de Domingo Faustino Sarmiento (1868-1874), fue levantado los días 15, 16 y 17 de setiembre de 1869 , en cumplimiento de la ley 302 de dicho año. Los resultados fueron aprobados por la ley 565 de 1872, con un leve aumento de las cifras para corregir el subregistro. El operativo siguió un conjunto de criterios que permiten caracterizarlo como el primer censo moderno de la Argentina. Dichos criterios incluyen: (i) la existencia de un organismo centralizado (la Oficina de Estadística Nacional, dependiente del Ministerio del Interior, creada en 1864 y disuelta en 1875); (ii) un diseño conceptual único; (iii) la voluntad de relevar de manera universal y simultánea a toda la población y, sobre todo, (iv) la ausencia de finalidades pre-estadísticas (por ejemplo fiscales y militares), propia de los relevamientos previos. A diferencia de los censos posteriores, el censo de 1869 fue realizado en un momento en que el Estado argentino no ejercía el control total de su territorio, razón por la cual existe una dualidad geográfica entre un territorio de soberanía teórica (áreas ocupadas por pueblos originarios), para la que sólo existen estimaciones inciertas sobre el número de habitantes, y un área de ocupación efectiva, en la que se aplicó el censo propiamente dicho (ver Figura 1). Conforme a los criterios fijados por los congresos internacionales de estadística del período, el censo de 1869 se basó en el recuento de la población de hecho (es decir, la población efectivamente presente al momento del relevamiento), realizado mediante entrevista directa (método Canvasser). ${ }^{4}$ El censo incorporó, además, a la población fluvial, el Ejército de Operaciones del Paraguay y una sumaria estimación de los argentinos en el exterior.

Como se verá más adelante, el diseño conceptual del censo incorporó tanto variables clásicas de este tipo de instrumento, como otras más novedosas atentas a la captación de poblaciones en situaciones críticas. El aspecto más cuestionado del censo remite a la escasa atención otorgada a las estructuras familiares y de hogar, rasgo común a los tres primeros censos nacionales $(1869,1895$ y 1914) levantados durante la llamada "estadística de autor" en la que predominó un enfoque estadístico esencialmente individual (Otero, 2006). Dadas las condiciones de la época, el nivel de cobertura puede ser considerado como muy satisfactorio para la parte del territorio que fue efectivamente censada (subregistro del orden del $4 \%$ contra el $30 \%$ de los relevamientos coloniales y de inicios del período independiente). ${ }^{5}$ Los resultados, conocidos en un tiempo bastante rápido para las condiciones de la época (1872), fueron ordenados de acuerdo a las jurisdicciones territoriales existentes (14 provincias y sus divisiones internas) ${ }^{6}$, distribuidas a su vez en cuatro regiones o "agrupaciones" (Este, Oeste, Norte y Centro). Esta clasificación, basada en criterios espaciales laxos y en la búsqueda de cierta simetría

\footnotetext{
${ }^{4}$ Elección que, a diferencia de otros sistemas como el householder norteamericano, otorgó un rol importante a los empadronadores elegidos entre los "vecinos más ilustrados, morales y activos, nacionales o extranjeros" con prioridad, cuando ello era posible, de los "empleados nacionales, provinciales (...), sacerdotes, maestros de escuelas, etc." (Argentina, 1872, p. 707). La preferencia por la entrevista directa derivó del alto grado de analfabetismo del período $(64,6$ y 78,1 \% de la población de 14 años y más para varones y mujeres, respectivamente).

5 La evolución del subregistro es analizada en Otero (2007: 207-211).

${ }^{6}$ En 1869, las 14 provincias se subdividían en 251 departamentos o partidos; si se suman otras áreas especiales, en general ubicadas en zonas de frontera, el número de unidades censales alcanza a 281.
} 
de los grupos definidos, se inspiraba en la geografía académica francesa del período y suponía, asimismo, un diagnóstico jerárquico a partir del cual interpretar la realidad argentina (Quintero, 2004).

A pesar de los vaivenes evocados, el censo de 1869 constituye la primera radiografía general de la Argentina independiente y tiene un valor que va más más allá del carácter puntual que esa fecha sugiere. La radiografía censal se inscribe en un momento particular, la presidencia de Domingo F. Sarmiento, la segunda de la llamada República unificada, tras la presidencia de Bartolomé Mitre (1862-1868). En tal sentido, constituye un momento de transición entre la larga etapa de guerras (desde las guerras de la Independencia y civiles hasta la secesión de la provincia de Buenos Aires de la Confederación entre 1852 y 1861), de devastadores efectos económicos, y la consolidación del Estado nacional a partir de 1880, caracterizada por la inmigración de masas, la expansión de las exportaciones y la infraestructura pública, entre otros rasgos. La importancia de la década del ochenta no debe hacer olvidar que durante las décadas de 1850 y 1860 ocurrieron también importantes cambios significativos, en términos institucionales y socio-económicos que hicieron posible el desarrollo posterior. Entre ellos se destaca el temprano avance de la urbanización (la población urbana alcanzó el $28,6 \%$ en 1869), fruto de migraciones internas de larga data y de la llegada de inmigrantes europeos. Los cambios económicos fueron igualmente relevantes aunque diferenciales según las provincias ya que coexistieron estructuras productivas tradicionales con economías más diversificadas en Córdoba, Tucumán y Cuyo, con actividades de mayor desarrollo capitalista en la región Litoral, entre las que se destacan las estancias de la pampa bonaerense, el boom lanero y el inicio de la agricultura del cereal en el sur de Santa Fe. ${ }^{7}$

\section{La medición de la calidad de vida}

Consideramos que la calidad de vida es "una medida de logro respecto de un nivel establecido como óptimo teniendo en cuenta dimensiones socioeconómicas y ambientales que dependen de la escala de valores prevaleciente en la sociedad y que varían en función de las expectativas de progreso histórico" (Velázquez, 2001, p. 15). De este modo, mientras la pobreza se mide con respecto a un piso, la calidad de vida se dimensiona respecto de un techo. Para estudiarla es habitual recurrir al análisis de dimensiones significativas, entre las que las condiciones de educación, salud y vivienda resultan insoslayables. Una jurisdicción que tuviera 50\% de alfabetos en 1869 , por ejemplo, estaría más cerca del techo (mejor situación relativa), mientras que, con ese mismo valor, en 1947 estaría más cerca del piso. Así se explica que en 1869 los pisos y techos de alfabetismo eran 0,0 y $73,1 \%$, respectivamente, mientras que en 1895 eran 0,0 y $81,5 \%$ y en 1947 eran 33,0 y $94,9 \%{ }^{8}$

7 Sobre el contexto del período, sobre el que no podemos extendernos aquí, ver Moreyra (1997) para los aspectos productivos y Otero (2006) para los demográficos.

8 Para 1914 no se dispone de datos de alfabetismo por departamento. Este censo muestra que la brecha entre provincias era de 33,3\% (Los Andes) a 82,2\% (Ciudad de Buenos Aires). 
Además de los estudios citados precedentemente, los trabajos de Barbeito y Lo Vuolo (1992); Bolsi et al (2006); Celemín, (2007); Connerly y Marans (1985); Delgado y Méndez (1996); Estés (1993); Halperín (1994); Longhi et al (2013); Sterimberg et al, (2004); Tanguay et al (2010); Torrado (2007); Velázquez (2016) y Wilkins (2003), entre otros, contribuyen a generar un marco apropiado que, como se verá más adelante, debe ser adaptado a las condiciones de vida y a la disponibilidad de información del período, tarea a la que se consagran las secciones que siguen. El índice resultante es un resumen de la calidad de vida que, conforme a la vocación comparativa del estudio, es susceptible de ser replicado para otros momentos históricos. Por último, las conclusiones analizan las principales implicancias de los resultados alcanzados.

\section{La educación en 1869}

Siguiendo una concepción cercana al moderno concepto de población de riesgo, el primer censo nacional buscó detectar a las personas en situaciones críticas, para lo cual incluyó datos sobre educación y salud. En lo relativo a la primera dimensión, que formaba parte de lo que en la época se definía como el "estado moral" de la población, la principal información que ofrece el censo es la de las personas que saben leer y escribir, es decir el alfabetismo, aunque medido de manera dicotómica y sin intentar determinar grados o niveles de conocimiento de la lecto-escritura. No se relevaron otros datos, como por ejemplo, los niveles de instrucción alcanzados. Sí fue relevada, en cambio, la población escolar'. Los resultados del censo arrojaron un total de 1.800 .000 habitantes, de los que sólo 312.000 sabían leer y escribir. Diego de la Fuente (Argentina, 1872, p. XXXVI), estimaba que la cifra real era $30 \%$ peor (218.000) y que la población menor de 6 años era de 316.000, por lo que la proporción de alfabetos era de aproximadamente $14,7 \%$.

La tasa mostraba considerable desigualdad entre las diferentes jurisdicciones del país (ver figura 2: alfabetización por cuartiles). En el mejor cuartil, la tasa supera al $19,02 \%$ de población alfabeta. El mejor valor se registra en la ciudad de Buenos Aires $(73,11 \%)$, mucho mayor al resto de las unidades. En general, los partidos cercanos a la principal ciudad del país tienen valores relativamente altos, destacando San Vicente con más del $40 \%$. Si establecemos un umbral del $30 \%$, el mismo es superado tan sólo por 12 unidades, la gran mayoría de ellas localizadas en la denominada Agrupación del Este, nueve en Buenos Aires, una en Santa Fe y una en Entre Ríos. Fuera de dicho agrupamiento, tan sólo la capital de San Juan se agrega a este selecto grupo. Dentro de este primer cuartil con mejor situación relativa, se destaca el amplio predominio de unidades bonaerenses, del sur de Santa Fe y del sur de Córdoba. La mayoría de las capitales provinciales también integran este grupo con mayor alfabetismo relativo (figura 2). En el otro extremo, es decir el cuartil con peor situación relativa, se encuentran unidades con menos del 8,13\% de población alfabeta. La situación más negativa es la de Anta (Salta) en donde la proporción de alfabetos es cero. En otras siete unidades

\footnotetext{
${ }_{9}$ La población escolar no fue incluida en el índice porque, además de estar afectada por la estructura demográfica, su influencia sobre la calidad de vida se verá reflejada en momentos posteriores, en este caso en la reducción del analfabetismo en el Censo de 1895.
} 
la proporción no llega al 3\%; cinco de ellas están situadas en Santiago del Estero (Agrupación del Centro), mientras que las dos restantes lo están en Tucumán y Salta (Agrupación del Norte). En general, las peores posiciones predominan ampliamente en el Norte, fundamentalmente en Santiago del Estero, Jujuy y Tucumán. También La Rioja y San Luis exhiben muy mala situación relativa en varios de sus departamentos. La mitad de las unidades $\left(2^{\circ}\right.$ y $3^{\circ}$ cuartil $)$ se halla comprendida en los valores intermedios de la distribución (entre 8,13 y 19,01\% de la población declara saber leer y escribir). Cabe recordar que el relevamiento censal no fue más allá del área mostrada en el mapa, por lo que las zonas que aparecen en blanco muy probablemente adolezcan de mayores deficiencias aún.

La heterogeneidad espacial del mencionado indicador no debe hacer olvidar que, vistos en conjunto, los niveles de alfabetización de la Argentina (lo mismo ocurría con la tasa de escolarización) estaban entonces entre los más altos de América Latina, superando a países como México o Brasil (Newland, 1997, p. 268). Dado que la organización del sistema público de enseñanza era aún muy incipiente (sus espectaculares logros son posteriores a este período), una parte sustantiva de la oferta educativa era de carácter privado (escuelas laicas, escuelas comunitarias de los grupos migratorios y escuelas confesionales, además de la alfabetización realizada en los hogares o por maestros particulares). Como sostiene Newland, la educación pública dependía de los estados provinciales y sólo marginalmente de la actividad municipal o nacional, lo que explica su mayor desarrollo en las provincias del Litoral que contaban con mayores recursos fiscales. Con todo, casos como el de San Juan sugieren que niveles de alfabetización relativamente altos podían alcanzarse por otras vías o a pesar de contar con menores recursos.

\section{La salud en 1869}

El censo releva asimismo información sobre las "condiciones especiales" de la población. Éstas incluyen un conjunto heterogéneo de variables, entre las que se destacan por su importancia diversas formas de discapacidad tanto física como mental: inválidos por accidente, inválidos por guerra, dementes, sordomudos, ciegos, cretinos/opas y población con bocio/coto. Las categorías mencionadas, que pueden ser vinculadas con géneros del período como las geografías médicas, constituían dolencias de impacto efectivo en la población, tanto en términos de salud como por su potencial efecto negativo sobre las actividades económicas o incluso el servicio de las milicias. A pesar de los problemas, la información censal brinda elementos de interés que permiten efectuar una aproximación a las condiciones de salud de la población, tanto más porque no existen datos de mortalidad para todas las provincias del país durante este período. Cabe consignar que las enfermedades incluidas en 1869 tuvieron algunas variaciones de interés durante el censo de 1895 y que, salvo la ceguera y la sordomudez, desaparecieron en el tercer censo nacional de 1914, reemplazadas por la genérica y no comparable categoría de "enfermo". ${ }^{10}$

\footnotetext{
${ }^{10}$ Sobre la evolución de la discapacidad en los censos argentinos, ver Pantano (1987).
} 
En el caso de la categoría inválidos, el censo discrimina entre inválidos por accidentes y por guerra. Los conflictos bélicos, endémicos durante toda la primera mitad del siglo XIX (guerras de la independencia y guerras civiles) continuaron con los enfrentamientos entre Buenos Aires y la Confederación; y alcanzaron su cénit con la mortífera Guerra de la Triple Alianza con el Paraguay (1864-1870), contemporánea a la realización del censo. Respecto de los inválidos por accidentes, el censo registra 3.308 casos, que representan al $0,21 \%$ de la población. El análisis territorial muestra que la proporción varía significativamente. El caso de mayor incidencia es San José (provincia de Santa $\mathrm{Fe}$ ) con el 13,1\% de su población afectada. Esta situación resulta totalmente anómala, dado que el segundo departamento con mayor proporción es Caldera (Salta), con 3,38\%. Un tercer caso supera al 2\%: el partido de Lincoln, en la provincia de Buenos Aires. Si se consideran las unidades con más del 1\%, se agregan otras dos: Belgrano (La Rioja) y Concepción del Uruguay (Entre Ríos). Si se amplía la escala de observación, existen dos áreas con mayor afectación relativa de este problema: las agrupaciones del Este del Oeste; la del Centro, por el contrario, muestra menor incidencia relativa. Resulta razonable conjeturar que las diferencias observadas no derivan exclusivamente de la ocurrencia de los eventos y que, quizás, reflejen también mejor atención médica en la zona central o, dicho de otro modo, que una proporción mayor de los accidentes ocurridos en esa región no quedaron debidamente registrados.

En relación a los inválidos por guerra, su magnitud ( 8.437 casos al sumar todas las unidades territoriales), casi triplica a los anteriores, y afecta al 0,53\% de la población. Cabe destacar que el informe censal minimiza esta cuestión, ya que su cuadro de síntesis incluye sólo 2.888 inválidos por guerra. La incidencia es mayor en el Noroeste Argentino, Mendoza y, sobre todo, Buenos Aires y el Litoral. Esta distribución, bastante diferente de la de los inválidos por accidente, se vincula con toda seguridad con el impacto de la Guerra del Paraguay (lugares de reclutamiento de los soldados, ubicación de las tropas de reserva y de los hospitales militares, etc.), contemporánea a la realización del censo. ${ }^{11}$ Existen, además, diferencias intra provinciales, fundamentalmente en beneficio de las capitales de provincia, que ostentan menores proporciones de población inválida por acciones bélicas, probablemente porque reclutaron menor proporción de población, porque los afectados gozaron de mejor atención médica o por una combinación de ambos factores. El departamento más afectado es Tres Arroyos (Buenos Aires) en donde $3,45 \%$ de su población se registra como inválida por la guerra, hecho que, al menos parcialmente, puede tener asociación con el avance militar de la frontera sur. Con más del 2\% de su población afectada se incluyen Tala (Entre Ríos) y Belgrano (La Rioja). La mayor proporción relativa de inválidos por guerra se registra en varios partidos del interior de Buenos Aires, San Luis, Jujuy y Mendoza. Por el contrario, Santa Fe, La Rioja, Santiago del Estero, ciudad de Buenos Aires y sus partidos más cercanos exhiben bajas proporciones. La mayor aleatoriedad de la distribución de inválidos por guerra se explicaría en el azar propio de las acciones militares, cuya composición refleja, a su vez, lugares de orígenes específicos de las tropas.

${ }^{11}$ Lamentablemente, el censo no proporciona la edad de los inválidos de guerra lo que hubiera permitido análisis más detallados sobre el particular. 
El censo registró también 3.674 habitantes como dementes, cifra que representa al $0,24 \%$ de la población del país. Aunque esta proporción sea baja, su incidencia es desigual a lo largo del territorio. El máximo registro $(3,86 \%)$ corresponde a Tuyú en la provincia de Buenos Aires. La barrera del 2\% es superada por Valle Grande (Jujuy), mientras que dos unidades más rebasan el $1 \%$ : Belgrano y San Martín (ambas en La Rioja). Más allá de estos casos extremos, la población demente tiene mayor incidencia en la Agrupación del Centro (especialmente en Córdoba y San Luis) y en la Agrupación del Norte (particularmente en Jujuy y Salta). Las proporciones también son altas en gran parte de Mendoza y norte de Santa Fe. Llama la atención un conglomerado de cinco partidos, relativamente cercanos a la ciudad de Buenos Aires (Moreno, Merlo, Las Heras, Matanza y San Vicente), que ostentan altos valores, lo que probablemente obedezca, al menos en parte, al envío de pacientes del interior a la Capital del país y su zona aledaña, regiones que contaban con instituciones de salud más avanzadas, hecho verificado en estudios de casos como el de la "Convalecencia porteña", institución abocada al cuidado de mujeres dementes (Pita, 2012). El área con menor proporción relativa de dementes es la Agrupación del Este, particularmente en los respectivos interiores de Buenos Aires y de Corrientes, aunque el primer caso también pueda verse afectado por el ya mencionado problema de la no distinción entre lugar de ocurrencia y lugar de residencia habitual de los enfermos. Fuera de esta agrupación también muestran bajas proporciones otras provincias como Santiago del Estero y Catamarca.

En cuanto a sordomudos, el censo registró 5.726 , o sea $0,38 \%$ de la población total. Su distribución sigue una pauta bastante clara: menor peso relativo en la Agrupación del Este, alto peso en las agrupaciones del Norte y, en menor medida, del Oeste. La Agrupación del Centro se encuentra, una vez más, en una posición intermedia. El análisis de los valores extremos nos permite detectar continuidades espaciales de gran interés. La mayor proporción de sordomudos se registra en Perico de San Antonio (Jujuy), donde está afectado el $4 \%$ de su población. La provincia norteña exhibe, además, un llamativo agrupamiento de cuatro departamentos contiguos (Valle Grande, Jujuy Capital, Perico del Carmen y el ya mencionado) en los que más del 3\% de su población padece este problema. Si se baja el piso al $2 \%$, se suman más departamentos contiguos de la provincia de Salta (Orán, San José de Metán y Candelaria). Fuera de este epicentro sólo aparece Guaymallén, en Mendoza. Un corte más, considerando ahora a los que superan el 1\%, sigue mostrando un abrumador predominio de los departamentos jujeños y salteños, a los que se agrega otro agrupamiento de unidades de Mendoza.

La incidencia de los ciegos (3.182 casos que representan al $0,20 \%$ de la población total), por su parte, es menor que la de sordomudos, pero su distribución resulta muy similar: menor peso relativo en la Agrupación del Este, alta proporción en la Agrupación del Norte, relativamente alta en el Oeste y relativamente intermedia en las provincias del Centro. Los valores extremos se registran en Belgrano e Independencia (ambas en La Rioja), unidades en las que los ciegos superan al 1\% de la población. Si se baja el umbral al 0,8\%, emerge un agrupamiento de tres departamentos riojanos, 
sumando San Martín a los dos precedentes. Por encima del 0,7 se agregan dos unidades más en Mendoza y Jujuy.

La categoría integrada por cretinos, estúpidos y opas resulta de particular interés. El hecho de que las distinciones entre estas enfermedades fueron objeto de arduos debates en las revistas especializadas durante el siglo XIX, sumado a su alta incidencia relativa, explica probablemente la creación de un grupo único por parte de los censistas. El cretinismo, por ejemplo, tendía en ocasiones a ser identificado con la idiocia (una forma de retardo mental). Las deficiencias mentales admitían, a su vez, diferencias de grado según la importancia del retraso (débiles mentales, imbéciles e idiotas; de menor a mayor gravedad, respectivamente). La denominación de opa, por su parte, refería a la población indígena o mestiza, es decir que incluía elementos de carácter étnico (Di Liscia, 2005). La distribución de este grupo (3.756 personas que representan el $0,24 \%$ de la población total) muestra mayor peso relativo en la Agrupación del Norte, sobre todo en las provincias de Salta y Jujuy, y relativamente alto en la del Oeste. Los valores extremos se registran en la Jujuy capital (2,11\% de su población). Si el umbral se fija en $1,5 \%$ se suman otros tres departamentos del Norte (Orán, Iruya y San José de Metán), que conforman un agrupamiento. Cuando el umbral se baja al 1\%, emergen otras 14 unidades, en el Norte (8), en el Oeste (5) y una en la provincia de San Luis. Las agrupaciones del Este y del Centro muestran, en general, menor proporción, salvo en sus periferias. Así, cerca de la ciudad de Buenos Aires se registran bajos valores, excepto en San Vicente, Matanza y Moreno. Las proporciones también son bajas en la provincia de Buenos Aires, con la excepción de Patagones en su extremo sur. En Entre Ríos y Corrientes, la magnitud de esta condición también es baja, con excepción de algunos partidos aislados en sus interiores.

Por último, el primer censo nacional registró 4.605 habitantes con bocio o coto que alcanzan al 0,30\% de la población. El bocio (o papera, para utilizar el término más frecuente en la actualidad) se caracteriza por una tumefacción de la parte delantera del cuello producida por el aumento de la tiroides. Su variada etiología incluye, entre otras, al bocio endémico producido por la escasez de yodo en la dieta. Dado que el hipotiroidismo durante la vida fetal podía producir cretinismo, es decir una forma particularmente grave de retraso mental, ambas enfermedades tendieron a ser confundidas en la época (Di Liscia, 2005), lo que debió impactar también en su captación estadística. La distribución, una vez más, afecta sobre todo a las provincias agrupadas en el Norte y Oeste, especialmente Mendoza. Precisamente el valor más extremo se localiza en el departamento de San Vicente (7,86\% de la población), en el oasis central mendocino. Por encima del 4\% sólo se suma Iruya, en Salta, mientras que si el umbral se baja al $3 \%$ aparecen ocho unidades (4 en el Norte y 4 en el Oeste). Resulta importante señalar que las capitales de Jujuy y de Mendoza integran este grupo. En la Agrupación del Centro el problema reviste escasa gravedad, salvo en San Luis. Lo mismo ocurre en el Este, con valores muy bajos en la ciudad de Buenos Aires y sus alrededores, provincia de Buenos Aires (excepto Tres Arroyos), Santa Fe y Entre Ríos. La única provincia afectada por este problema dentro del Este es Corrientes, incluso en su propia capital provincial. 
Si se agrupan todas las anteriores en una categoría denominada población con enfermedades (ver figura 3), procedimiento que permite reducir los problemas de captación evocados para cada una de ellas, la cifra alcanza a las 20.943 personas, que representan al 1,31\% de la población total. Desde luego, algunas personas podían tener más de una enfermedad, dato no informado por el censo, lo que no modifica sustancialmente los resultados. Conforme a los análisis parciales, este grupo tiene mayor peso relativo en las jurisdicciones del Norte y del Oeste. En el centro, los valores son intermedios, mientras que en el Este, y especialmente en torno de la ciudad de Buenos Aires, son bajos. La mayor proporción de población con enfermedades se registra en San Vicente (Mendoza), con 11,44 \% de su población afectada. Por encima del $8 \%$ se agregan tres departamentos de Jujuy. Superan la barrera del 6\% otros 10 departamentos de las provincias de Jujuy, Salta, La Rioja y Mendoza. Recién se agrega una unidad en el Este (Tuyú) al bajar el umbral al 3\% de la población. La composición de este grupo, si se considera este último nivel, es de 31 unidades del Norte y Oeste y una del Este. Por el contrario, si se parte de las mejores situaciones, por debajo de $0,2 \%$ hay cinco casos en el Este, dos en el centro y sólo uno en el Norte. En estos últimos casos, además, es posible que la explicación esté vinculada en parte con mayores niveles de subregistro.

Otro indicador censal de la dimensión salud, médicos, es obtenida de la lista de profesiones, y medida habitualmente por el número de ellos cada 1.000 habitantes, dato que sólo se halla disponible para la escala provincial (ver figura 4). Su distribución refleja una vez más la posición claramente mejor del Litoral y muy en particular la provincia de Buenos Aires, donde se concentraban alrededor de la mitad de los egresados (Newland, 1997, p. 272). De modo análogo a lo observado para la educación, la medicina científica convivía de manera complementaria con otras formas de atención de las necesidades, como la medicina familiar y popular y con otras figuras que, sin ser médicos, contaban con alguna experiencia (por ejemplo, facultados, flebótomos y boticarios) ya que el proceso de medicalización (creación de instituciones, difusión de terapéuticas y remedios científicos, persecución al curanderismo, etc.) impulsado desde las instancias gubernamentales aún no había comenzado de manera sistemática, sobre todo a escala nacional. Con todo, el indicador resulta pertinente para su comparación con censos posteriores y porque permite detectar diferencias espaciales consistentes con otras dimensiones analizadas. ${ }^{12}$

\section{La vivienda en 1869}

Además de la población, el primer censo nacional de 1869 relevó las viviendas, simultaneidad que, con algunas variantes, caracterizará a la tradición censal argentina. La importancia de esta empresa se agiganta, si se tiene en cuenta que la vivienda, por su carácter material y por su relativa facilidad de captación, es una de las dimensiones que mejor refleja los niveles de riqueza y bienestar de la sociedad. El censo distinguió cuatro tipos de viviendas, sobre la base de los materiales del techo (azotea, teja, madera

\footnotetext{
${ }^{12}$ Para un análisis en detalle de la distribución de los médicos en 1869 y de las discusiones sobre el particular, ver Rodríguez et al (2013).
} 
y paja) subdivididos, a su vez, según el número de cuerpos o pisos. En todo el país sólo había 346 casas de azotea de tres pisos, que representan un escasísimo 0,14\% del total de viviendas. Conforme a lo esperable, su distribución estaba restringida a la ciudad de Buenos Aires, Belgrano y alrededores, más algunos otros partidos de las provincias de Buenos Aires y Entre Ríos. Se registra también algún caso excepcional en jurisdicciones de Córdoba y Corrientes. La mayor proporción $(2,73 \%)$ se localizaba en Merlo, provincia de Buenos Aires. De manera previsible, las casas de azotea de dos pisos eran más numerosas (2.915), pero de todos modos bastante excepcionales $(1,20 \%$ del total) y su distribución seguía un patrón similar al precedente: mayor concentración en ciudad de Buenos Aires y alrededores, norte de la provincia de Buenos Aires, algunos departamentos de Entre Ríos, sur de Santa Fe y la capital de Corrientes. Fuera de la Agrupación del Este, las viviendas de este tipo sólo se destacan en las capitales provinciales de Córdoba y San Juan. La mayor proporción corresponde a la provincia de Buenos Aires (12\% en el partido de Lincoln). Por último, las casas de azotea de un cuerpo contabilizaban 41.259 (16,95\% del total de viviendas). La mayor parte se concentraba, una vez más, en la ciudad de Buenos Aires, norte y extremo sur de la provincia homónima, sur de Entre Ríos y Santa Fe; se agrega, fuera de la Agrupación del Este, una alta proporción relativa en el Oeste (particularmente en San Juan y Mendoza). El mayor peso de estas viviendas se localiza en San Juan capital $(89,81 \%)$ y en el vecino partido de Trinidad (85\%). Además de los casos ya mencionados, sólo superan la barrera del 70\% la ciudad de Buenos Aires y Belgrano. Por encima del $60 \%$ se agrega Patagones, en el pionero extremo sur de Buenos Aires. Finalmente, por encima del 50\% se agregan Flores y Bahía Blanca (Buenos Aires), Pocito y San Martín (muy cercanos a la capital de San Juan) y dos departamentos en La Rioja cercanos a la capital provincial. En la mayoría de los departamentos, sin embargo, este tipo de vivienda no alcanza a representar al 3\% del total.

Las casas de teja, por su parte, eran muy escasas en todo el territorio censado y prácticamente no tienen impacto en términos proporcionales (había tan sólo 178 casas de teja de dos pisos, que representan un insignificante $0,07 \%$ del total). Este puñado de viviendas estaba más representado en las capitales de Salta y Jujuy, además de los departamentos de Goya (Corrientes) y Colón (Entre Ríos). Además de factores ligados a la estructura social (peso de las familias ricas de antigua data en las provincias del norte, por ejemplo) su distribución puede derivar también de tradiciones de construcción propias de cada región y del tipo de materiales disponibles, como lo sugiere, por ejemplo, la distribución de las viviendas de madera, mucho más frecuentes en las provincias de Corrientes, Entre Ríos y Buenos Aires. Las casas de teja de un piso eran 7.423 (3,05\% del total) y tenían mayor peso relativo en el Norte (Salta, Jujuy, Tucumán) y, en la Agrupación del Este, sólo en Corrientes. Precisamente, la mayor proporción de estas viviendas $(89,52 \%)$ se registra en la capital de Corrientes. Hay que bajar el piso hasta $40 \%$ para que se integre la capital de Salta, mientras que por encima del $30 \%$ se agregan Caa-Catí en Corrientes y las capitales de Jujuy y La Rioja. Estas distribuciones ponen de manifiesto otro aspecto importante: la vivienda, a diferencia de otros indicadores, constituye una riqueza acumulativa y transmisible de una generación a otra. Por esta 
razón las regiones de asentamiento más temprano tienen, necesariamente, un parque habitacional más valioso que no se correlaciona necesariamente con otras dimensiones de calidad de vida, como lo sugiere la mayor concentración de viviendas de mejor construcción en el Oeste del país.

Si agrupamos las casas de azotea y de teja (ver figura 5), es decir las viviendas de mejor calidad, existían 52.121 unidades $(21,41 \%$ del total). Esta categoría tiene mayor peso relativo en la ciudad de Buenos Aires y alrededores, norte de la provincia de Buenos Aires, sur de Santa Fe y en los ejes fluviales de Entre Ríos y Corrientes. Fuera de la Agrupación del Este tienen también alto peso relativo en el Oeste (especialmente en Mendoza y San Juan) y en la zona central de Salta (Norte). Los valores más altos se registran en la capital de Corrientes (100\%), proporción que, dada su magnitud, debe ser considerada con mucha precaución; por encima del $90 \%$ se agrega la capital de San Juan. Si se supera el $80 \%$, se suman el vecino departamento de Trinidad (San Juan) y la ciudad de Buenos Aires. Por arriba del $70 \%$ se destaca también el contiguo partido de Belgrano y por encima del 60\% se agrega el vecino partido de Flores y Patagones. Por último, la barrera de 50\% la superan también Pocito y San Martín (que forman un agrupamiento junto a la capital de San Juan), la capital de Salta y un departamento en La Rioja. En la Agrupación del Centro, por el contrario, la proporción de viviendas de materiales más sólidos es muy baja, salvo en la capital de Córdoba.

En 1869 había, además, 1.208 casas de madera de dos cuerpos (0,50\% del total), que tenían mayor peso relativo en la ciudad de Buenos Aires y alrededores (San Martín, Conchas y Tigre), costa atlántica de Buenos Aires (Ajó y Tuyú) y algunos partidos del interior de Entre Ríos y Corrientes. Fuera del Este, casi no existen este tipo de viviendas, prueba de que la disponibilidad de materiales era un elemento decisivo en un período caracterizado por las dificultades de transporte. Las casas de madera de un piso son, desde luego, mucho más abundantes: 19.453 (7,99\% del total). Su mayor peso relativo está en el interior de Corrientes, Santiago del Estero (zona forestal) y La Rioja. La mayor proporción fue censada en Loreto (Santiago del Estero), en donde 99,95 \% de las casas era de madera. Por encima del $90 \%$ se agrega San Luis (Corrientes). Superando al $80 \%$ se suma Guasayán (Santiago del Estero) y Famatina (La Rioja). Por arriba del $70 \%$ se incluyen, además, San Cosme (Corrientes) y al superar el 50\% se agregan Empedrado, Itatí y Lomas (Corrientes), que forman una suerte de conglomerado; lo mismo ocurre con Salavina y Soconcho en Santiago del Estero.

Por último, las casas de paja, grupo que reúne a las viviendas más precarias y que remite a la clásica imagen del rancho de adobe, ilustran de modo elocuente las condiciones de vida de la mayoría de la población argentina en el Primer Censo Nacional. Este tipo de viviendas alcanzaba entonces 170.682 unidades que representan un abrumador 70,11\% del total de viviendas. Su distribución espacial resulta elocuente si se tiene en cuenta que en la mitad de los departamentos del país, la proporción de este tipo de casas supera al $83 \%$ del total. La peor situación relativa se registraba en la Agrupación del Centro (San Luis, Córdoba, Santiago del Estero), ya que en gran parte de sus unidades superan al $97 \%$ de las unidades. También tienen alto peso en el Norte 
Velázquez y Otero. Calidad de vida por departamentos, provincias y regiones en el primer censo nacional (1869)

(Tucumán, Salta, Jujuy). En la Agrupación del Este, por el contrario, este tipo de casas tiene, en general, menor peso relativo. Las excepciones las constituyen el norte de Santa $\mathrm{Fe}$, el este de Corrientes y el sur de Buenos Aires.

Vistas en conjunto, las distribuciones analizadas evocan una situación de precariedad material en prácticamente todo el país. Como lo ha mostrado Liernur (1993), para Buenos Aires, entre mediados del siglo XIX y la gran transformación posterior a la década de 1880, la ciudad se caracterizó por su carácter precario, efímero, transitorio o fugaz, aspecto que es más visible en la fotografía, el catastro y los censos de población que en otros registros históricos. Esa "ciudad efímera", según sus palabras, es perceptible en las casas de madera y chapa de los barrios populares, desde las casillas familiares hasta los conventillos, pero también en edificios y construcciones más complejas y se hará más evidente con la gran expansión urbana y la inmigración de masas de la década de 1880. Según Liernur, la precariedad de infraestructuras se tradujo en la alta frecuencia de incendios y en las condiciones socio-sanitarias que favorecieron la gran epidemia de fiebre amarilla de 1871. La Argentina que emerge del censo de 1869 mezcla, en suma, dos formas de precariedad en términos de vivienda: por un lado, la de la ciudad efímera impulsada por el vertiginoso crecimiento económico y demográfico de la que Buenos Aires sería el ejemplo paradigmático. Por otro lado, una precariedad que es previa al proceso expansivo y que, a diferencia de la anterior, se asocia más a una pobreza de medios materiales de carácter más permanente y con mayor impacto en los ámbitos rural y del interior del país, aunque también es perceptible en las grandes urbes. La mejor situación relativa de Buenos Aires fue claramente percibida por De la Fuente quien, además de constatar que esa jurisdicción tenía "más casas de azotea que todos los otros estados reunidos", conjeturó que si se incluyeran variables como el valor y la extensión de las propiedades, las casas de azotea y teja "representarían cuatro veces más que todas las de igual clase de toda la república” (Argentina, 1872, pp. IL-L). Según el mismo comentarista, esa desproporción se acrecentaría en el siguiente censo en razón del mayor crecimiento de la provincia.

El censo registró 1.596 .975 individuos en viviendas y 243.464 casas en total, por lo que para el territorio censado el cálculo indica 6,56 personas por vivienda (ver figura 6). Recordemos la debilidad de los tres primeros censos nacionales para captar hogares y familias. Aunque existen indicadores sumarios como el número de personas por vivienda, esta medida puede ser considerada una aproximación indirecta al grado de hacinamiento (en el caso de que el tamaño de las viviendas no difiera significativamente, supuesto no del todo realista a la luz de los análisis previos). Por otra parte, debe tenerse presente que el hacinamiento tiene una variabilidad cultural e histórica, ya que su percepción como problema y su operatividad técnica depende del contexto de cada sociedad. A título de ejemplo, el umbral que define el hacinamiento es muy diferente según se trate de sociedades actuales o pre-transicionales, es decir con altos niveles de fecundidad como la que retrata el censo de 1869. Cualquiera sea el caso, la cantidad de personas por vivienda es muy desigual a lo largo del territorio, algo que resulta más perceptible a nivel departamental (los promedios provinciales no dan cuenta de esto). 
Más que diferencias de fecundidad, cuya disminución es posterior al período aquí analizado, la cantidad de personas por vivienda refleja probablemente la presencia de agregados en las unidades censales, ${ }^{13}$ diferencias en la complejidad de las estructuras familiares $\mathrm{y}$, sobre todo, los niveles de pobreza y el impacto del proceso migratorio, tanto interno como europeo, que implica cierto grado de hacinamiento residencial, al menos durante los años iniciales de instalación de los inmigrantes. Ello explicaría el alto grado de hacinamiento en la ciudad de Buenos Aires y Barracas al Sud. Por otra parte, como lo sugieren los datos de Torrado (2007, p. 216) sobre la ciudad de Buenos Aires en 1869, la cantidad de miembros por vivienda tenía asociación estrecha con la situación socio-económica ya que iba desde 4,9 personas, en la sección 20 (conformada por sectores acomodados) a 7,9 personas en la sección 1 (habitada por sectores modestos). La ciudad como un todo, por su parte, ostentaba un promedio de 6,1. Si las observaciones sobre el caso porteño fueran generalizables al total del país, podría postularse que el mayor hacinamiento del interior obedecería, al menos en parte, a su mayor pobreza relativa. El hacinamiento es bajo en el interior de la provincia de Buenos Aires, salvo en Tordillo y Tuyú. Por el contrario, se destaca alto hacinamiento en dos partidos del interior de Corrientes y en Lomas, periferia de su capital provincial. Esta presencia de la población más pobre en la periferia de la ciudad, probablemente, contribuya a explicar los buenos indicadores que ostenta la capital correntina en el rubro calidad de las viviendas. En síntesis, el hacinamiento en la Agrupación del Este se presenta en lugares muy puntuales. En el resto del país, el hacinamiento resulta alto en el Norte, Centro y Oeste, en general en los respectivos interiores provinciales, probablemente más asociado con mayor proporción relativa de agregados y de familias extendidas y compuestas. Visto el país como un todo, las situaciones más críticas se presentan en Barracas al Sud (15,24 individuos por vivienda). Al superar la barrera de los 12 individuos por vivienda, se agrega Ancaste en Catamarca. Por encima de los 10 se incorporan dos departamentos en Corrientes (Goya y Curuzú Cuatiá), uno en Jujuy (Ledesma, vinculado con la agroindustria azucarera) y uno en Córdoba (Pocho). En el extremo opuesto, es decir menor hacinamiento, se encuentran los partidos de Bahía Blanca, Tres Arroyos y Lincoln (en la frontera bonaerense), Pila y Salto (Buenos Aires) y Paso de los Libres en Corrientes.

\section{El índice de calidad de vida en 1869}

Las variables de educación, salud y vivienda reseñadas hasta aquí permiten, a partir de un índice, construir una imagen de las condiciones de vida de la población argentina hacia 1869. Variables cuya distribución resulta coincidente en algunos casos y, en otros, no. Las coincidencias y diferencias pueden explicarse por la naturaleza de las

\footnotetext{
${ }^{13}$ Como lo postulan Cacopardo y Moreno (1997, p.19), el termino agregado remite a un "concepto muy abarcativo y (...) de carácter residual" ya que podía incluir situaciones muy diversas como "peones y jornaleros, huérfanos, recogidos, parientes lejanos, domésticos, aprendices y hasta transeúntes". Este trabajo, basado en el microanálisis de las listas nominativas del censo, constituye la única aproximación a las estructuras familiares de 1869 pero, lamentablemente, solo incluye las provincias del interior (Catamarca, Córdoba, Salta, Santiago del Estero y Corrientes) lo que impide comparaciones para el total del país.
} 
variables que tienden a perjudicar o beneficiar más a la población de algunos territorios, y que el índice recoge. El índice deriva de un compromiso entre la información disponible, las unidades de análisis elegidas y el intento de aproximación a la escala de valores de la sociedad en cada momento histórico. Resultaría inaceptable, en la actualidad, un índice que no contemple los aspectos ambientales del bienestar, dimensión que para la Argentina de 1869 no fue considerado porque no resultaba aún relevante, ni era captada por el sistema estadístico. Otro criterio que se suma para la elección de los componentes consiste en incorporar variables que permitan comparaciones de largo plazo con miras a los períodos más recientes.

Por todo ello, nuestra propuesta para estimar la calidad de vida en 1869 incluye las variables que se informan detalladas en la Tabla 1. Allí puede observarse que las variables tienen distinta amplitud total. Así, algunas varían entre 0 y $100 \%$, mientras que otras lo hacen entre 0,49 y 0,04 por mil, como ocurre con los médicos por habitantes. Otro elemento a enfatizar es que algunas son variables de costo, dado que su incremento implica peor situación relativa, tal es el caso de la población con enfermedades y el hacinamiento. Otras, por su parte, son variables de beneficio, dado que su incremento se corresponde con mejores situaciones relativas, y son la tasa de alfabetismo, los médicos por mil habitantes y las viviendas de azotea y teja. La integración de estas tasas fue efectuada mediante su transformación en números-índice parciales, en los cuales los valores extremos se transforman entre 1 y 0 para reflejar la mejor y la peor situación relativa, respectivamente.

Para variables de costo (población con enfermedades y personas por vivienda):

Indice $=\frac{\text { Máximo }-a}{\text { Máximo - mínimo }} \stackrel{\text { territorial })}{\text { donde a la variable de costo especifica e la unidad }}$

Así, por ejemplo, Ledesma (Jujuy) tenía 10,35 personas por vivienda, cifra que llevada a número-índice da 0,40 (lo que ubica a Ledesma más cerca de la peor situación relativa o máximo que del mínimo cuyo valor es 3,14 ).

Para variables de beneficio (tasa de alfabetismo, médicos/1000 habitantes, viviendas de azotea y teja):

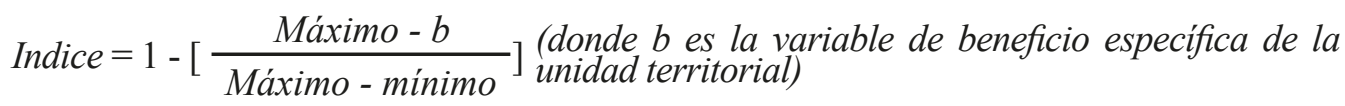

Así, por ejemplo, Tandil (Buenos Aires) tenía una tasa de alfabetismo de 19,3 $\%$. Esta tasa, llevada a número-índice es 0,26 (lo que ubica a Tandil más cerca de la peor situación relativa o mínimo que del máximo, que es 73,11$)$.

La fórmula para el cálculo del ICV resulta de: 


\begin{tabular}{l}
\hline \multicolumn{1}{c}{ Índice de Calidad de Vida } \\
\hline ( Índice Analfabetismo) x 2 \\
+ Índice Población con Enfermedades \\
+ Índice Médicos \\
+ Índice Viviendas azotea y teja \\
+ Índice Personas/vivienda
\end{tabular}

Así el ICV para Tandil es:

\begin{tabular}{|c|c|c|c|c|c|c|c|c|c|c|c|c|c|}
\hline 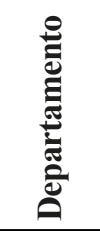 & 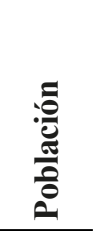 & 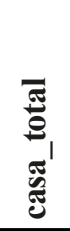 & 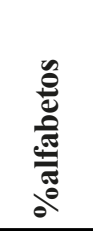 & 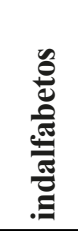 & 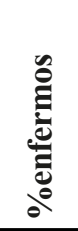 & 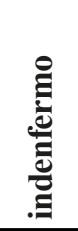 & 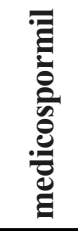 & 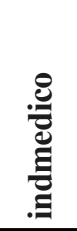 & 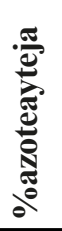 & 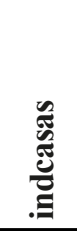 & 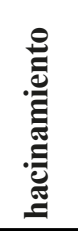 & 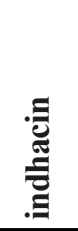 & ${ }_{0}^{\infty}$ \\
\hline Tandil & 4870 & 888 & 19,30 & 0,26 & 0,47 & 0,96 & 0,49 & 1,00 & 5 & 0,05 & 5,48 & 0,81 & 5,57 \\
\hline
\end{tabular}

El mapa síntesis del índice propuesto (ver figura 7) muestra que la calidad de vida de la población argentina, en 1869, era muy inequitativa a lo largo del territorio. La mejor situación relativa se registra en la ciudad de Buenos Aires y los partidos cercanos, con la excepción de Barracas al Sud, Matanza y Merlo que se ubican en un segundo escalón. En el resto de la Agrupación del Este, todos los partidos de la provincia de Buenos Aires se ubican en el primer o en el segundo cuartil; lo mismo ocurre en Entre Ríos y Santa Fe. En Corrientes, en cambio, sólo su capital se posiciona en el primer cuartil, mientras que la mayoría de sus departamentos lo hace en el segundo y cinco de ellos, ubicados en los extremos norte y sur de la provincia, en el tercero. Los restantes agrupamientos se encuentran en situaciones mucho más desfavorables. El caso más extremo es la Agrupación del Norte, en la cual todos sus departamentos se encuadran en las peores condiciones de vida, con las excepciones de las capitales provinciales de Tucumán y Salta y el departamento de Santa Bárbara en Jujuy, que logran ubicarse en el tercer escalón de calidad de vida. La Agrupación del Oeste, por su parte, muestra situaciones contrapuestas: el oasis central sanjuanino exhibe buenas condiciones, el de Mendoza lo hace en menor medida, al igual que la capital de Catamarca. El resto de los territorios provinciales se posiciona dentro de los cuartiles tres o cuatro del índice. Por último, la Agrupación del Centro muestra bastante diversidad. Por un lado, el sur de Córdoba, al igual que su capital y la cabecera de San Luis, se posicionan en el segundo escalón de calidad de vida. Por el contrario, la gran mayoría de los departamentos de Santiago del Estero se ubican en los últimos.

La brecha entre la mejor y la peor situación es alta. El ranking es encabezado por la Ciudad de Buenos Aires (8,92 puntos), mientras que la peor situación relativa la sufre Ledesma (Jujuy) que alcanza sólo 1,99 puntos. La Tabla 2 y la Tabla 3 suministran los valores de las 14 capitales provinciales, las 14 provincias y las cuatro agrupaciones establecidas en el censo y permiten apreciar que la inequidad era mayor entre las 
Velázquez y Otero. Calidad de vida por departamentos, provincias y regiones en el primer censo nacional (1869)

capitales provinciales que entre las provincias, hecho afectado sin duda por el alto ICV de la ciudad de Buenos Aires.

\section{Conclusiones}

Como lo muestra de modo consistente este análisis geográfico, histórico y multidimensional, las desigualdades en la Argentina durante el primer censo nacional de 1869 eran altas, tanto entre las grandes regiones, como entre las provincias y entre las unidades menores. A continuación, resumimos las brechas del ICV entre las peores y mejores situaciones en los distintos niveles de desagregación propuestos al inicio del trabajo. Si se consideran las regiones, la diferencia entre la mejor y peor situación relativa (Agrupaciones del Este y del Norte, respectivamente) es de 3,03 puntos; a escala de provincias (Buenos Aires y Jujuy), la amplitud aumenta a 4,32 puntos y entre los departamentos (Ledesma en Jujuy y ciudad de Buenos Aires), la brecha trepa a 6,93 puntos. A pesar de excepciones en San Juan, Córdoba, norte de Entre Ríos y en Corrientes, la mayor desigualdad de los departamentos no alcanza a desdibujar el predominio de la Agrupación del Este que casi duplica los valores de las otras tres.

Vista la calidad de vida en términos de las unidades menores, los análisis realizados muestran otros dos elementos de interés. El primero es el impacto de la urbanización, ya que los índices de calidad de vida tienden a ser mayores en las ciudades que en los interiores provinciales. El segundo, la influencia de la expansión de la frontera, ya que dejando de lado las zonas de asentamiento más temprano, como la ciudad de Buenos Aires y su hinterland o sus equivalentes santafesinos, los indicadores favorables de las provincias del Litoral pueden ponerse en relación con los procesos de expansión de la frontera interior, causa clave, por otra parte, del aumento de la riqueza en esas zonas. La relevancia de estos resultados no radica sólo en su novedoso abordaje desde múltiples dimensiones, con enfoque interdisciplinario y distinta desagregación espacial, desde luego, sino también, en su consistencia con los resultados parciales y las hipótesis sobre la Argentina del período que se han vertido en otros estudios pero, sobre todo, en el grado de precisión suplementaria que permite aportar a las discusiones ya que, como bien sostiene Hora $(2010,154)$, "la información disponible [para el período 1850-1880] sobre temas fundamentales como el bienestar y la equidad resulta pobre e incompleta, y sólo permite formular algunas apreciaciones superficiales".

Además de la desigualdad observada a diferentes niveles, el impacto de la urbanización y la influencia de la expansión de la frontera, la segunda conclusión importante de este trabajo remite al plano metodológico y consiste en destacar la validez de los instrumentos (especialmente el ICV y los SIG) así como de las fuentes utilizadas (sobre todo los censos nacionales). En efecto, la potencialidad operativa de los Sistemas de Información Geográfica, sobre la que no hace falta insistir aquí, permite recuperar fuentes históricas de notable riqueza como los censos de población decimonónicos que, a pesar de sus reiteradas visitas, constituyen una cantera importante de información. Esta primera radiografía de la calidad de vida argentina, la más antigua disponible hasta el presente, 
muestra asimismo la potencialidad de los censos de población históricos para el estudio de dimensiones que van mucho más allá de su utilización exclusivamente demográfica.

Lamentablemente, no se dispone de fuentes análogas para períodos previos, lo que hubiera permitido comparaciones retrospectivas de gran interés; sabiendo que los indicadores utilizados pueden ser definidos como incrementales (es decir que, salvo crisis de enorme magnitud, no deberían experimentar retrocesos) la calidad de vida debería tender a aumentar con el transcurso del tiempo. El debate no radica, desde luego, en esa constatación, sino más bien en saber si las diferencias entre unidades espaciales tendieron incrementarse, disminuir o mantenerse estables. La respuesta a este interrogante queda en manos de trabajos posteriores. En particular, la comparación de la calidad de vida de 1869 con la del segundo censo nacional de 1895, nos permitirá medir con precisión los niveles de heterogeneidad y equidad espacial del indicador después de un período caracterizado por la notable expansión de las exportaciones, de la riqueza y el proceso de consolidación del Estado Nacional, tema de fuerte debate en los estudios de historia económica.

Resta, asimismo, indagar estadísticamente respecto de los factores de diferenciación que contribuyen a explicar mejor los mapas propuestos (urbanización, escala urbana, estructura demográfica, procesos migratorios, estructura económica y social, decisiones políticas, centralidad y accesibilidad, riqueza generada, etc.) tarea que, al igual que la evolución de largo plazo del índice de calidad de vida, no podemos realizar aquí y que será objeto de los futuras investigaciones.

\section{Referencias Bibliográficas}

Argentina (1872). Primer Censo de la República Argentina verificado en los días 15, 16 y 17 de setiembre de 1869 bajo la dirección de Diego G. de la Fuente. Buenos Aires: Imprenta del Porvenir.

Barbeito, A. \& Lo Vuolo, R. (1992). La modernización excluyente. Transformación económica y Estado de Bienestar en Argentina. Buenos Aires: UNICEF/ CIEPP/LOSADA.

Bolsi, A., Longhi, F. \& Paolasso, P. (2006). "E1 Norte Grande Argentino entre el progreso y la pobreza". Población \& Sociedad, 12-13, 227-266.

Cacopardo, M.C. (1967). República Argentina, cambios en los límites nacionales, provinciales y departamentales a través de los censos nacionales de población. Buenos Aires: Instituto Torcuato Di Tella.

Cacopardo, M.C. \& Moreno, J.L. (1997). "Cuando los hombres estaban ausentes: la familia del interior de la Argentina decimonónica”. En H. Otero \& G. Velázquez (comp.), Poblaciones Argentinas. Estudios de Demografia diferencial (pp. 1328). Tandil: CIG-IEHS.

Camargo Mora, M. G. (ed.) (1996). III Seminario Latinoamericano de Calidad de Vida Urbana. Mérida, Universidad de los Andes: Instituto de Geografía. 
Velázquez y Otero. Calidad de vida por departamentos, provincias y regiones en el primer censo nacional (1869)

Celemín, J. P. (2007). "El estudio de la calidad de vida ambiental: definiciones conceptuales, elaboración de índices y su aplicación en la ciudad de Mar del Plata, Argentina". Hologramática, 1(7), 71-98.

Connerly, C. \& Marans, R. (1985). "Comparing Two Global Measures of Perceived Neighborhood Quality". Social Indicators Research, 17, 29-47, doi: dx.doi. org/10.1007/BF00354111

Delgado, M. T. \& Méndez, E. (1996). Planificación territorial. Medio ambiente y calidad de vida. Mérida: Litorama.

Di Liscia, M. S. (2005). "Relaciones peligrosas: sobre bocio, cretinismo e inferioridad (Argentina, 1870-1920)". En C. Agostoni \& E. Speckman Guerra (ed.), De normas y transgresiones. Enfermedad y crimen en América Latina (1850-1950) (pp. 21-54). México, Universidad Nacional Autónoma de México: Instituto de Investigaciones Históricas.

Estés, R. (1993). "Hacia un índice de calidad de vida: enfoques empíricos para la evaluación del bienestar humano a nivel internacional". En Kliksberg, B (ed.), Pobreza, un tema impostergable (pp. 132-148). Caracas: FCE-PNUD, UNESCO.

Gelman, J. (coord.) (2011). El mapa de la desigualdad en la Argentina del siglo XIX. Rosario: Prohistoria Ediciones.

Gelman, J.; Santilli, D. (2018). "Wages and standards of living in the 19th century from a comparative perspective. Consumption basket, Bare Bone Basket and welfare ratio in Buenos Aires, 1825-1849". Investigaciones de Historia Económica, 14, 94-106. doi.org/10.1016/j.ihe.2016.09.001.

González Bollo, H. (2014). La fábrica de las cifras oficiales del Estado argentino, 1869-1947. Bernal: Universidad Nacional de Quilmes.

Guzmán, T. (2012). "El plano de una ciudad desigual. La distribución espacial de la riqueza en la ciudad de Buenos Aires en 1839". Quinto Sol, 16 (1), 1-27.

Halperín, L. (1994). Condiciones de vida de la población de Mar del Plata 1992-1994. Mar del Plata: Facultad de Humanidades, Universidad Nacional de Mar del Plata.

Hora, R. (2010). Historia económica de la Argentina en el siglo XIX. Buenos Aires: Siglo XXI Editores.

Liernur, J. F. (1993). "La ciudad efímera. Consideraciones sobre el aspecto material de Buenos Aires: 1870-1910”. En: Liernur, J \& Silvestri, G. El umbral de la metrópoli. Transformaciones técnicas y cultura en la modernización de Buenos Aires (1870-1930) (pp. 177-222). Buenos Aires: Sudamericana.

Longhi, F., Bolsi, A., Velázquez, G., Paolasso, P. \& Celemín, J.P. (2013). "Fragmentación socio-territorial y condiciones de vida en Argentina en los albores del siglo XXI". Revista Latinoamericana de Población, 7(12), 99-131.

Lucero, P., Mikkelsen, C., Sabuda, F., Ares, S., Aveni, S. \& Ondartz, A. (2007). "Calidad de vida y espacio: una mirada geográfica desde el territorio local”, Hologramática, 1(7), 99-125.

Massé, G. (2003). Historia demográfica argentina, 1869-1914. Versión digital de los tres primeros censos nacionales. [CD-ROM]. Buenos Aires: Instituto Nacional de Estadísticas y Censos. 
Moreyra, B. (1997). “Agricultura y Ganadería (1850-1880)”. En Nueva historia de la Nación Argentina, VI: La configuración de la República independiente (1810-c. 1914). Buenos Aires: Academia Nacional de la Historia, 65-100.

Newland, C. (1997). "Enseñanza elemental y superior (1810-1862)". En Nueva historia de la Nación Argentina, VI: La configuración de la República independiente (1810-c. 1914). Buenos Aires: Academia Nacional de la Historia, 261-275.

Míguez, E. (2008). Historia económica de la Argentina. De la conquista a la crisis de 1930. Buenos Aires: Sudamericana.

Mikkelsen, C., Ares, S., Sabuda, F. \& Lucero, P. (2013). "Calidad de vida urbana en la Argentina de la postconvertibilidad. Procesos sociales y territoriales en el período 2003-2012". En XII Jornadas Argentinas de Estudios de Población. Bahía Blanca.

Olave, F. D. \& Bodini, H. (1995). Metodología básica para detectar calidad de vida en ciudades intermedias. Chillán: Departamento de Geografía, Universidad del Bío-Bío.

Otero, H. (2004). "La transición demográfica argentina a debate. Una perspectiva espacial de las explicaciones ideacionales, económicas y político-institucionales”. En $\mathrm{H}$. Otero (dir.). El mosaico argentino. Modelos y representaciones del espacio y de la población, siglos XIX-XX (pp. 71-170). Buenos Aires: Editorial Siglo XXI.

Otero, H. (2006). Estadística y nación. Una historia conceptual del pensamiento censal de la Argentina Moderna. Buenos Aires: Prometeo.

Otero, H. (2007). “Censos antiguos, 1869, 1895, 1914, 1947”. En Torrado, S (comp.). Población y bienestar en la Argentina del primero al segundo Centenario. Una historia social del siglo XX. Buenos Aires: EDHASA, Tomo I, 187-213.

Pantano, L. (1987). La discapacidad como problema social. Reflexiones y propuestas. Buenos Aires: EUDEBA.

Pita, V. (2012). La casa de las locas. Una historias social del Hospital de Mujeres Dementes. Buenos Aires, 1852-1890. Buenos Aires: Prohistoria.

Quintero, S. (2004). "La interpretación del territorio argentino en los primeros Censos Nacionales de Población (1869, 1895, 1914)". En Otero, H (dir.). El mosaico argentino. Modelos y representaciones del espacio y de la población, siglos XIXXX (pp. 267-297). Buenos Aires: Editorial Siglo XXI.

Recchini, Z. \& Lattes, A. (ed.) (1975). La población de Argentina. Buenos Aires: CICRED. Rodríguez, M.L., Carbonetti, A. \& Andreatta, M. (2013). "Prácticas empíricas y medicina académica en Argentina. Aproximaciones para un análisis cuanticualitativo del Primer Censo Nacional (1869)". Historia Crítica, (49), 81-108., doi: 10.7440/ HISTCRIT49.2013.05

Salvatore, R. (1998). "Heights and Welfare in Late-Colonial and Post-Independence Argentina”. En J. Komlos y J. Baten (eds). The Biological Standard of Living in Comparative Perspective. Stuttgart: Franz Steiner Verlag, 97-121.

Santilli, D (2016). “¿Por qué un dossier sobre desigualdad, distribución y nivel de vida en el S XIX?" Folia Histórica del Nordeste, 26, 64-72.

Sterimberg, E., Sánchez, C., Cuervo De Forero, A. \& Ramírez, J. (2004). "Diseño de un sistema de indicadores socioambientales para el distrito capital de Bogotá". Serie estudios y perspectivas. Santiago de Chile: Cepal - Naciones Unidas. 
Tanguay, G., Rajaonson, J., Lefebvre, J. \& Lanoie, P. (2010). "Measuring the sustainability of cities: A survey-based analysis of the Use of Local Indicators". Ecological Indicators, 10(2), 407-418.

Torrado, S. (2007). Población y bienestar. La Argentina del primero al segundo centenario. Buenos Aires: Edhasa.

Velázquez, G. (2001). Geografía, calidad de vida y fragmentación en la Argentina de los noventa. Análisis regional y departamental utilizando SIG. Tandil: Centro de Investigaciones Geográficas, Universidad Nacional del Centro de la Provincia de Buenos Aires.

Velázquez, G. (2008). Geografía y bienestar. Situación local, regional y global de la Argentina luego del censo de 2001. Buenos Aires: EUDEBA.

Velázquez, G. (2016). Geografia y calidad de vida en Argentina. Análisis regional y departamental (2010). Tandil: IGEHCS-CIG.

Velázquez, G. \& García, M. (1999). Calidad de vida urbana. Aportes para su estudio en Latinoamérica. Tandil: Centro de Investigaciones Geográficas, Universidad Nacional del Centro de la Provincia de Buenos Aires.

Velázquez, G., Mikkelsen, C., Linares, S. \& Celemín, J.P. (2014). Calidad de vida en Argentina. Ranking del bienestar por departamentos (2010). Tandil: Centro de Investigaciones Geográficas, Universidad Nacional del Centro de la Provincia de Buenos Aires.

Wilkins, H. (2003). "The need for subjectivity in EIA: discourse as a tool for sustainable development". Environmental Impact Assessment Review, 23(4), 401-414, doi: dx.doi.org/10.1016/S0195-9255(03)00044-1. 


\section{ANEXOS: TABLAS Y FIGURAS}

Figura 1. División provincial. Argentina, 1869.

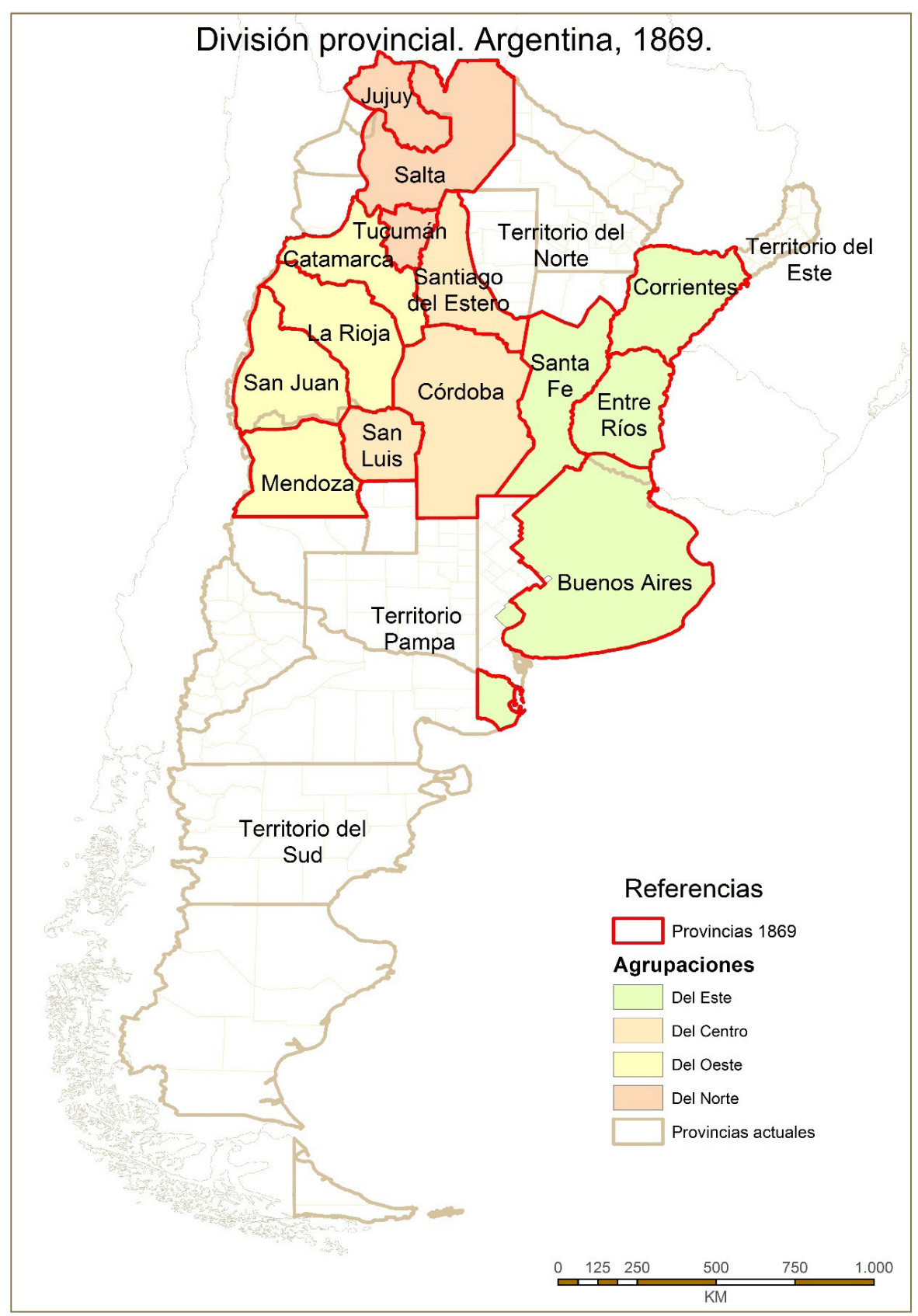

Fuente: elaboración del autor usando SIG y datos del Censo Nacional 1869 
Figura 2. Población que sabe leer y escribir. Argentina, 1869.

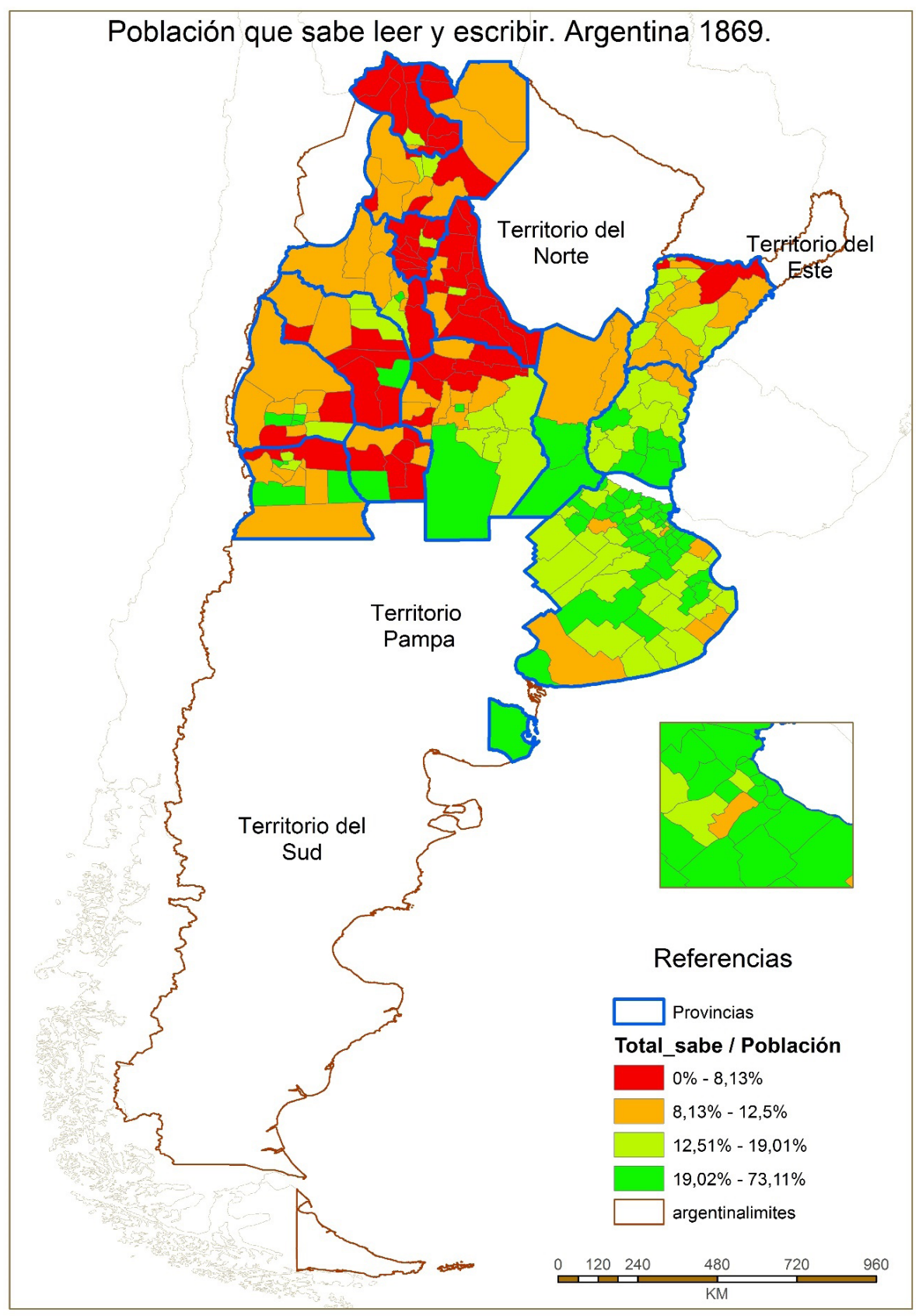

Fuente: elaboración del autor usando SIG y datos del Censo Nacional 1869 
Figura 3. Población con enfermedades. Argentina, 1869.

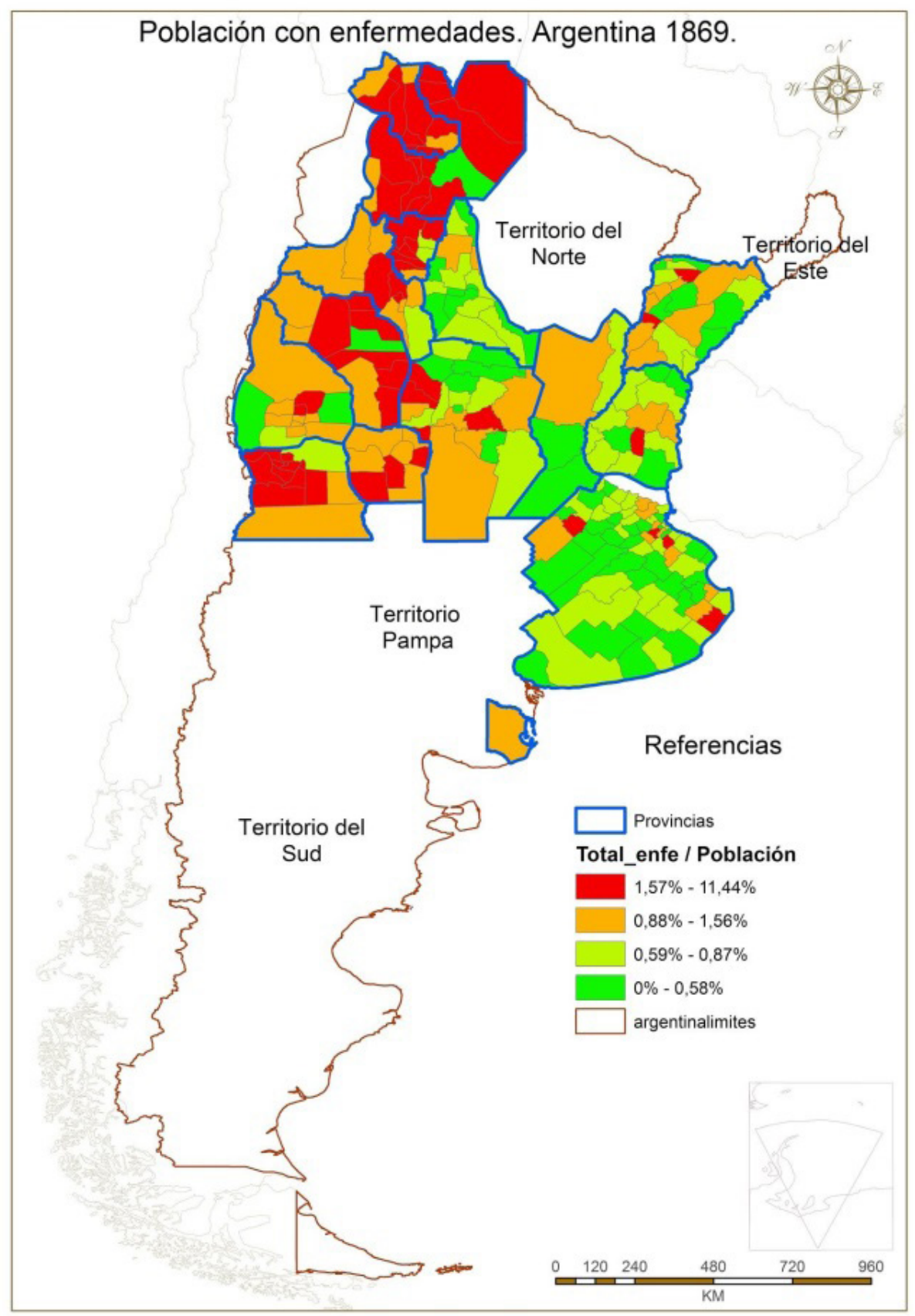

Fuente: elaboración del autor usando SIG y datos del Censo Nacional 1869 
Figura 4. Médicos/1000 habitantes. Argentina, 1869.

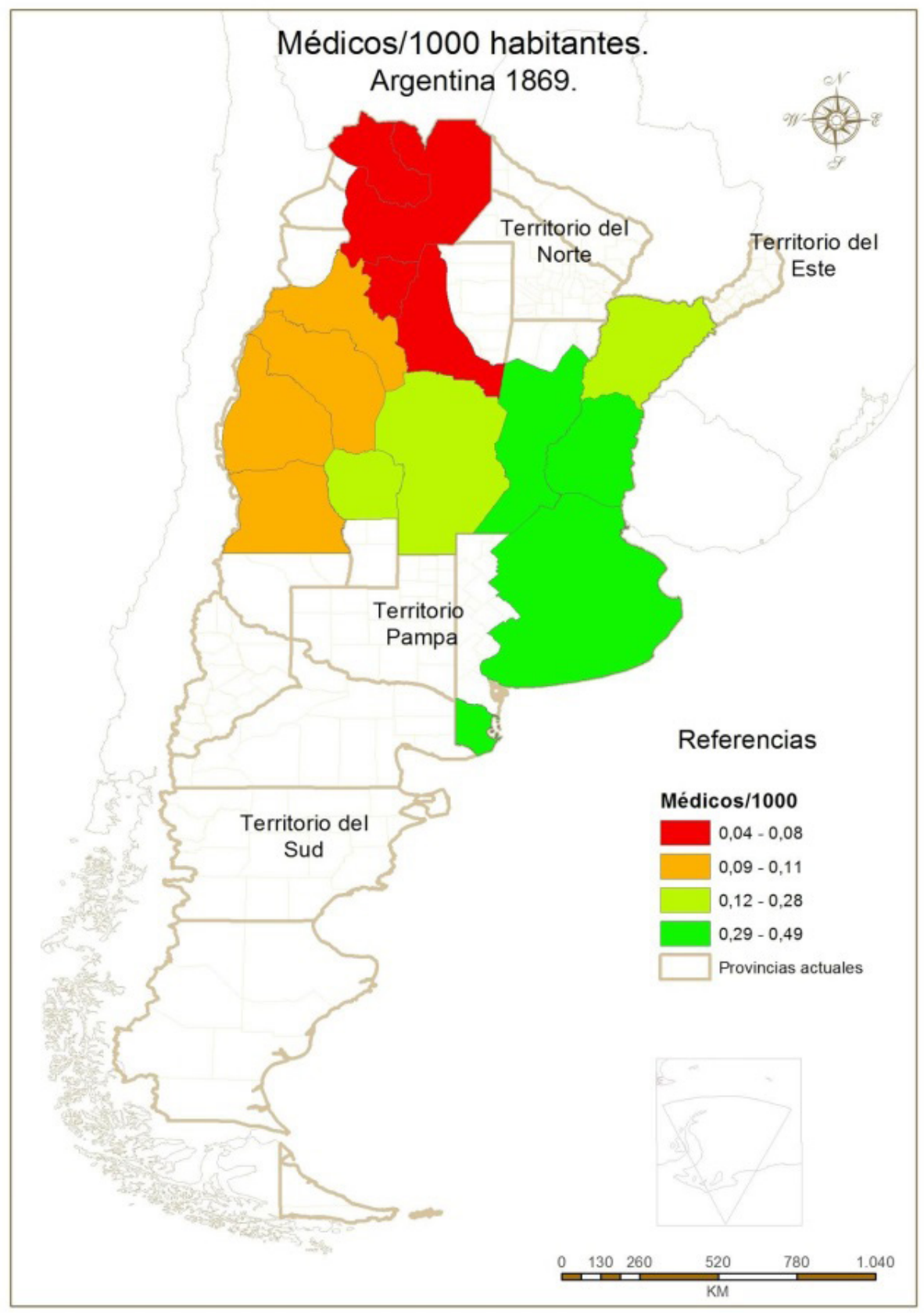

Fuente: elaboración del autor usando SIG y datos del Censo Nacional 1869 
Figura 5. Casas de azotea y teja. Argentina, 1869.

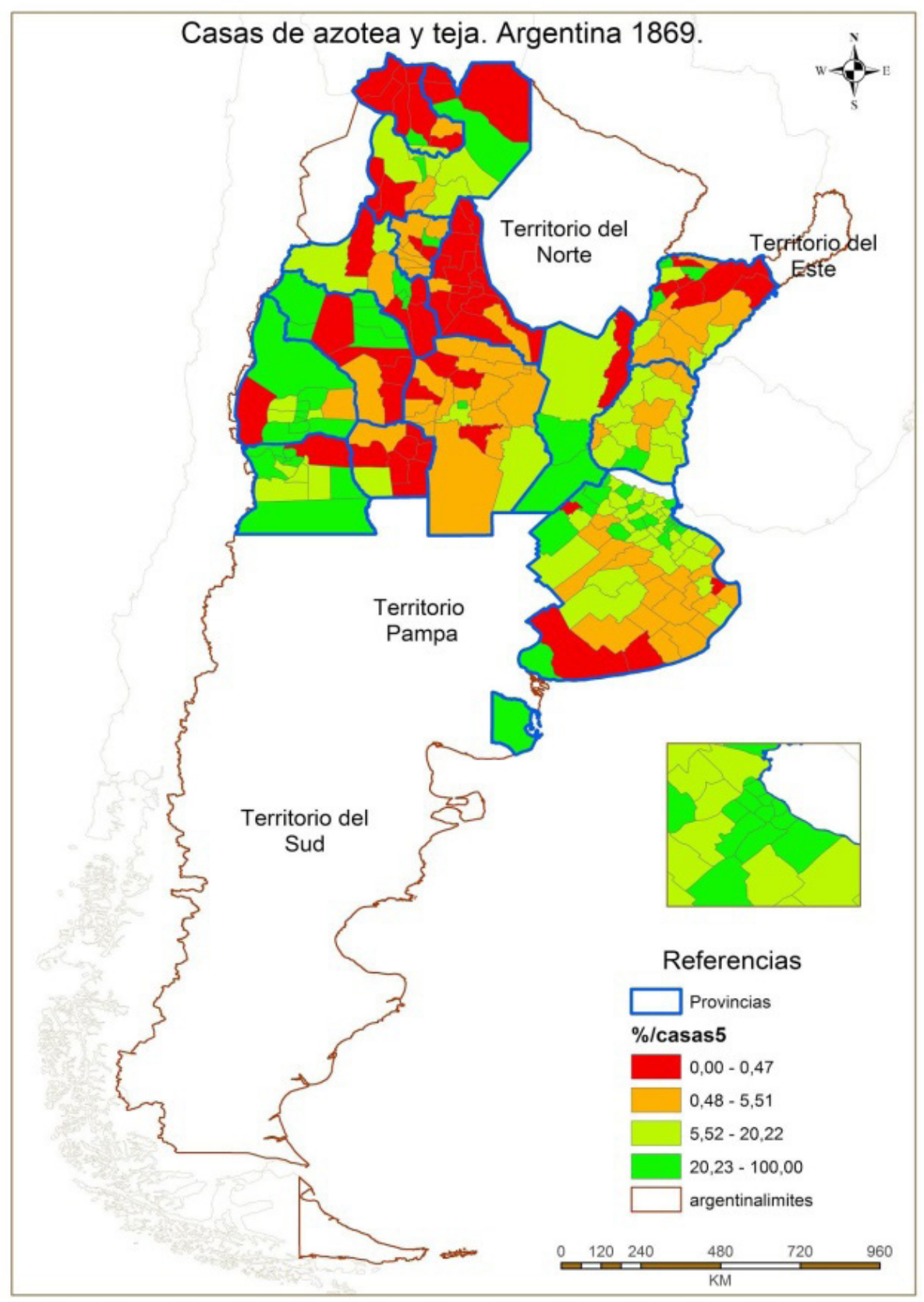

Fuente: elaboración del autor usando SIG y datos del Censo Nacional 1869 
Figura 6. Personas por vivienda. Argentina, 1869.

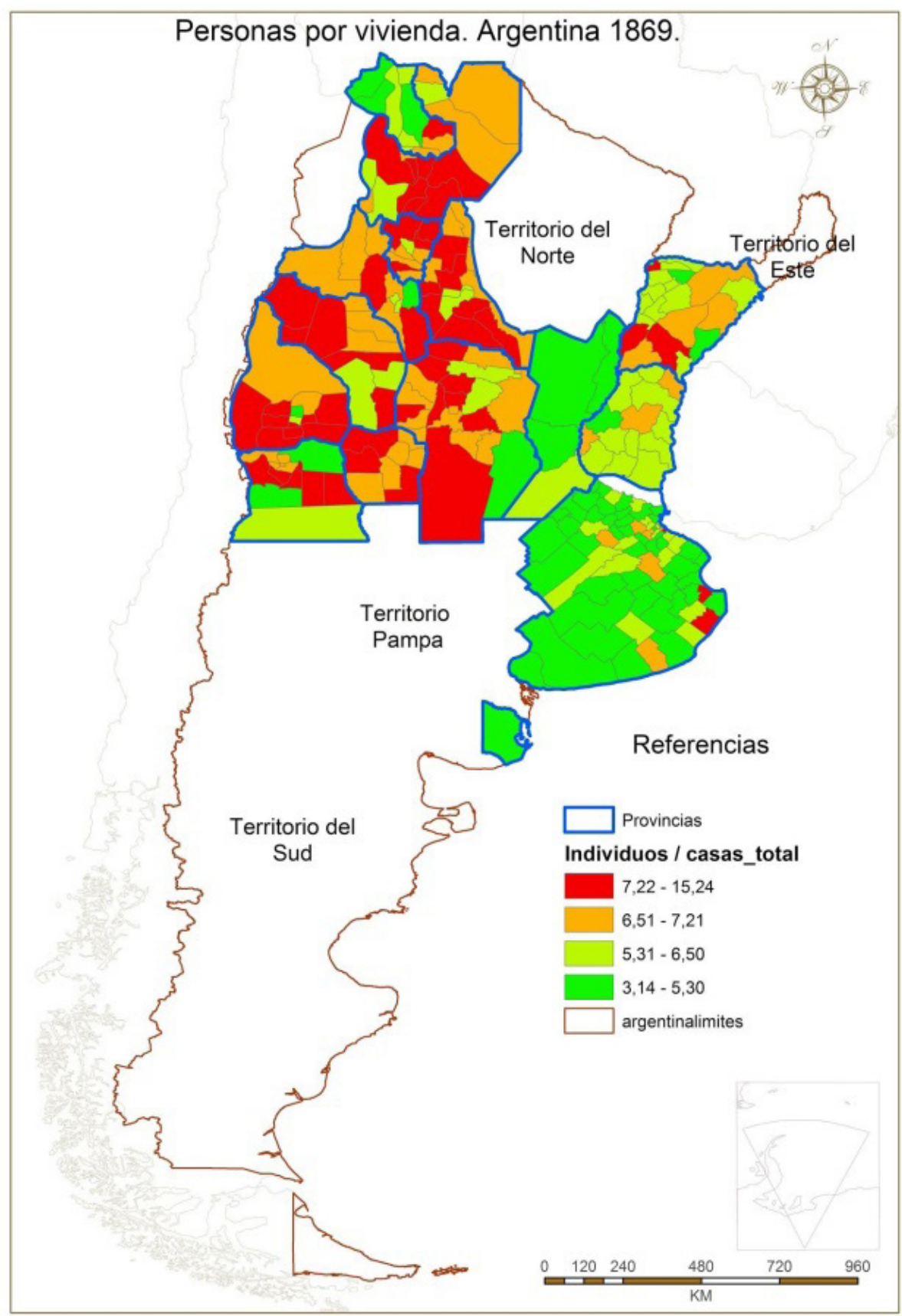

Fuente: elaboración del autor usando SIG y datos del Censo Nacional 1869 
Figura 7. Índice de calidad de vida. Argentina, 1869.

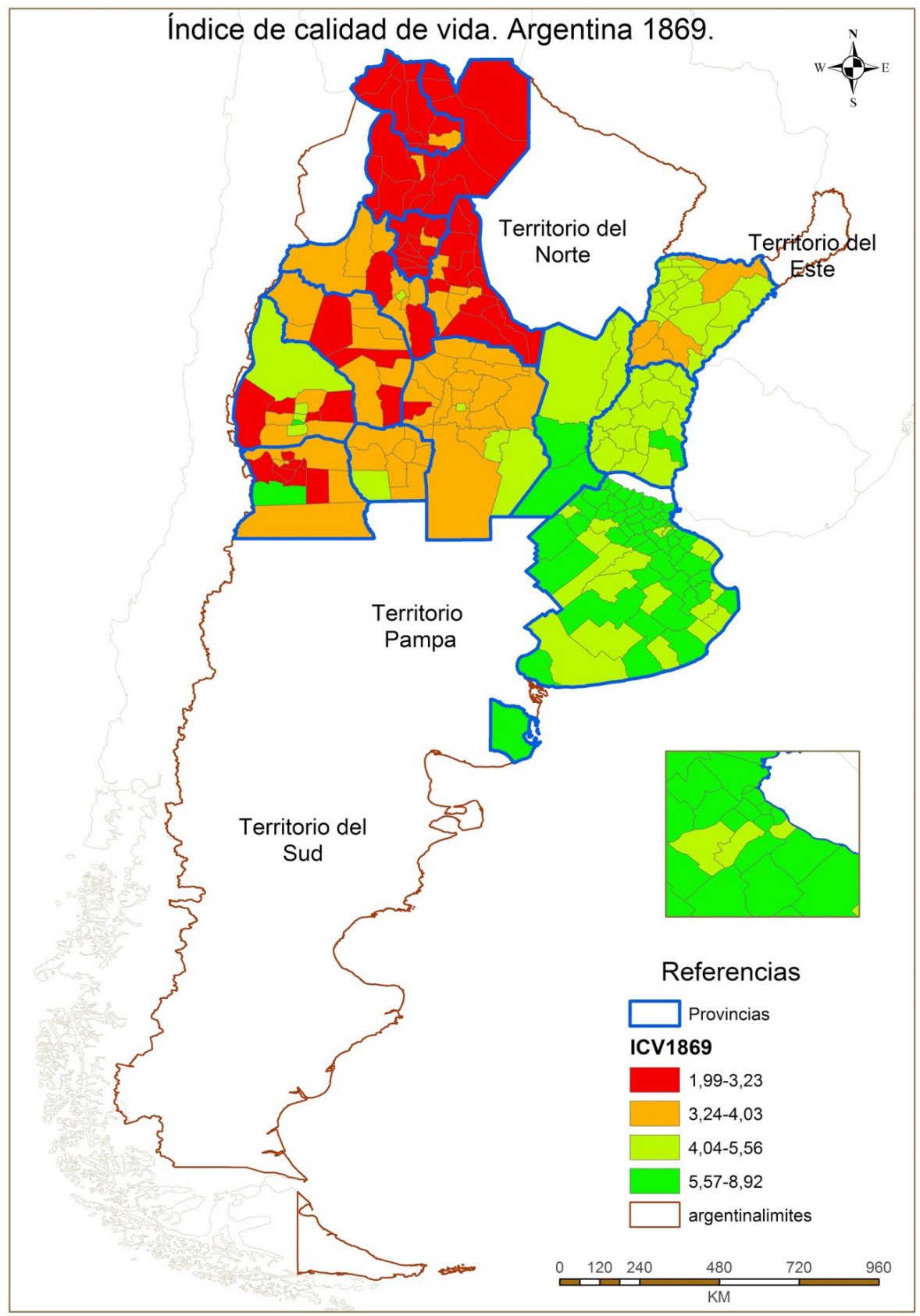

Fuente: elaboración del autor usando SIG y datos del Censo Nacional 1869 
Tabla 1. Dimensiones y variables del Î́ndice de Calidad de Vida (ICV). Argentina, 1869.

\begin{tabular}{|c|c|c|c|}
\hline Dimensión & $\begin{array}{c}\text { Peso } \\
\text { relativo }\end{array}$ & Variables (ponderación final) & $\begin{array}{c}\text { Valores extremos } \\
\text { (Máximo - mínimo) } \\
\end{array}$ \\
\hline Educación & $1 / 3$ & Tasa de alfabetismo (total $1 / 3$ ) & $(73,11-0)$ \\
\hline \multirow[b]{2}{*}{ Salud } & \multirow[b]{2}{*}{$1 / 3$} & 1/2 Población con enfermedades (total 1/6) & $(11,44-0)$ \\
\hline & & $\begin{array}{l}\text { 1/2 Médicos/1000 hab. (total } 1 / 6 \text {, escala } \\
\text { provincial) }\end{array}$ & $(0,49-0,04)$ \\
\hline \multirow[t]{2}{*}{ Vivienda } & \multirow[t]{2}{*}{$1 / 3$} & $\begin{array}{l}1 / 2 \text { Proporción de viviendas de azotea y } \\
\text { teja (total } 1 / 6)\end{array}$ & $(100-0)$ \\
\hline & & $1 / 2$ Personas por vivienda (total $1 / 6$ ) & $(15,24-3,14)$ \\
\hline
\end{tabular}

Fuente: elaboración del autor sobre la base del censo de 1869, Argentina (1872)

Tabla 2. Índices de calidad de vida (ICV) de capitales provinciales y provincias. Argentina 1869.

\begin{tabular}{llll}
\hline \multicolumn{1}{c}{ Capitales provinciales } & ICV & \multicolumn{1}{c}{ Provincias } & ICV \\
\hline Ciudad de Buenos Aires & 8,92 & Buenos Aires & 6,96 \\
\hline Santa Fe & 6,59 & Santa Fe & 5,60 \\
\hline Corrientes & 6,19 & Entre Ríos & 4,96 \\
\hline San Juan & 6,16 & San Juan & 4,62 \\
\hline Paraná (Entre Ríos) & 5,40 & Corrientes & 4,43 \\
\hline Córdoba & 5,33 & Córdoba & 3,95 \\
\hline San Fdo.del Valle de Catamarca & 4,50 & San Luis & 3,71 \\
\hline San Luis & 4,32 & Catamarca & 3,53 \\
\hline Salta & 3,97 & La Rioja & 3,52 \\
\hline Mendoza & 3,93 & Mendoza & 3,23 \\
\hline La Rioja & 3,92 & Santiago del Estero & 3,09 \\
\hline San Miguel de Tucumán & 3,91 & Tucumán & 3,30 \\
\hline Santiago del Estero & 2,94 & Salta & 2,99 \\
\hline San Salvador de Jujuy & 2,72 & Jujuy & 2,64 \\
\hline
\end{tabular}

Fuente: elaboración del autor sobre la base del censo de 1869, Argentina (1872) 
Tabla 3. Población e Índices de calidad de vida (ICV) según regiones. Argentina, 1869.

\begin{tabular}{|c|c|c|c|}
\hline \multicolumn{2}{|c|}{ Regiones censales } & \multirow{2}{*}{$\begin{array}{r}\text { Población } \\
823.552\end{array}$} & \multirow{2}{*}{$\frac{\text { ICV }}{6,12}$} \\
\hline & Agrupación del Este & & \\
\hline Buenos Aires & & 491.487 & 6,96 \\
\hline Corrientes & & 129.023 & 4,43 \\
\hline Entre Ríos & & 133.573 & 4,96 \\
\hline \multirow[t]{2}{*}{ Santa Fe } & & 69.469 & 5,60 \\
\hline & Agrupación del Centro & 358.355 & 3,65 \\
\hline Córdoba & & 198.823 & 3,95 \\
\hline San Luis & & 45.538 & 3,71 \\
\hline \multirow[t]{2}{*}{ Santiago del Estero } & & 113.994 & 3,09 \\
\hline & Agrupación del Oeste & 220.416 & 3,65 \\
\hline Catamarca & & 71.293 & 3,53 \\
\hline La Rioja & & 42.536 & 3,52 \\
\hline Mendoza & & 64.063 & 3,23 \\
\hline \multirow[t]{2}{*}{ San Juan } & & 42.524 & 4,62 \\
\hline & Agrupación del Norte & 198.776 & 3,09 \\
\hline Jujuy & & 30.761 & 2,64 \\
\hline Salta & & 67.781 & 2,99 \\
\hline Tucumán & & 100.234 & 3,30 \\
\hline
\end{tabular}

Fuente: elaboración del autor sobre la base del censo de 1869, Argentina (1872) 